\title{
Soft, comfortable polymer dry electrodes for high quality ECG and EEG recording
}

\author{
Yun-Hsuan Chen ${ }^{1,2 *}$, Maaike Op de Beeck ${ }^{1}$, Luc Vanderheyden ${ }^{3}$, Evelien Carrette ${ }^{4}$, \\ Vojkan Mihajlović ${ }^{5}$, Kris Vanstreels ${ }^{1}$, Bernard Grundlehner ${ }^{5}$, Stefanie Gadeyne ${ }^{4}$, \\ Paul Boon ${ }^{4}$,Chris Van Hoof ${ }^{1,2}$
}

1 imec, Kapeldreef 75, 3001, Heverlee, Belgium; E-Mails: Yun-Hsuan.Chen@imec.be (Y.C.); Maaike.OpdeBeeck@imec.be(M.O.); Kris.Vanstreels@imec.be(K.V.); Chris.VanHoof@imec.be(C.V.)

2 Dept. of Electrical Engineering, KU Leuven, Kasteelpark Arenberg 10, 3001 Heverlee, Belgium

3 Datwyler Sealing Solutions, Industrieterrein Kolmen 1519, 6570 Alken, Belgium; E-Mail: luc.vanderheyden@datwyler.com (L.V.)

4 Dept. Neurology, Ghent Univ. Hospital, 1K12IA De Pintelaan 185, 9000 Gent, Belgium; E-Mails: evelien.carrette@UGent.be (E.C); Stefanie.Gadeyne@UGent.be (S.G); paul.boon@uzgent.be (P.B)

5 Holst Centre/ imec-nl, High Tech Campus 31, 5656 AE Eindhoven, the Netherlands; E-Mails: Vojkan.Mihajlovic@imec-nl.nl (V.M.); Bernard.Grundlehner@imec-nl.nl (B.G.)

* Author to whom correspondence should be addressed; E-Mail: Yun-Hsuan.Chen@imec.be (Y.C.); Tel.: +32-1628-3574; Fax: +32-1628-9400.

\begin{abstract}
Conventional gel electrodes are widely used for biopotential measurements despite important drawbacks such as severe discomfort, skin irritation and very long set-up time. Dry electrodes with metal pins overcome most of these problems, however, their rigidity causes discomfort and pain. Flexible polymer-based dry electrodes with high user comfort are presented in this paper, fabricated from EPDM rubber containing various additives for optimum conductivity, flexibility and fabrication yield. The impedance of those electrodes is measured on phantoms and human skin. Polymer electrodes with optimum composition show contact impedances only $\sim 10$ times larger than gel electrodes, and are easily capable of recording strong biopotential signals such as ECG. For low-amplitude signals, such as EEG, the electrodes need to be coupled with a small active circuit. EEG recordings using active dry electrodes connected to a clinical EEG recording system show very promising results: alpha-waves can be observed clearly when subjects close their eyes, and correlation and coherence analyses reveal high similarity between dry and gel electrode signals. Also, all subjects reported that our polymer electrodes were not causing discomfort.
\end{abstract}


To conclude, the polymer-based dry electrodes are promising alternatives for the rigid dry electrodes in development and the conventional gel electrodes.

Keywords: flexible polymer dry electrode; material optimization; impedance; high quality biopotential recordings; ECG; EEG; high user comfort; conductive polymer.

\section{Introduction}

Wet gel electrodes are widely used in biopotential recordings. The gel improves the electrode skin contact and hydrates the skin, resulting in high quality signal recording with limited motion artifact disturbances. However, the drawbacks of the gel are also well known. During recording, signal degradation will occur due to gel drying, and skin irritation is often observed. For EEG, the electrode set-up is time-consuming and has to be done by an expert. After EEG recording, electrode removal is difficult, often even painful, and time consuming [1].

To avoid these drawbacks of wet electrodes, dry electrodes are introduced. Commercially available dry electrode recording systems are equipped with metal-coated or metal electrodes [2]. The metal layer might flake off during usage and the rigidity of full metal electrodes causes pain during recording. The flexible polymer-based dry electrodes presented in this paper overcome the drawbacks of wet gel and of hard metal dry electrodes. These dry electrodes were fabricated using flexible conductive polymer materials. Various additives are added into the polymer, type and quantity was optimized to obtain low contact impedance and good mechanical properties for better comfort and skin contact. The conductive polymer electrodes were used for ECG and EEG recording, and the obtained biopotential signals were compared to those of conventional wet electrodes, by looking at correlation, coherence and signal to noise (SNR) ratio, following the protocol introduced in [3].

\section{Experimental Section}

\subsection{Dry electrode fabrication based on conductive polymer}

Various (conductive) additives are mixed in an ethylene propylene diene monomer (EPDM) matrix, followed by compression molding, see Fig. 1 [4]. Carbon is used as conductive additive.

Figure 1: various electrodes after shaping in a pin-shaped mold to obtain comb-like electrodes

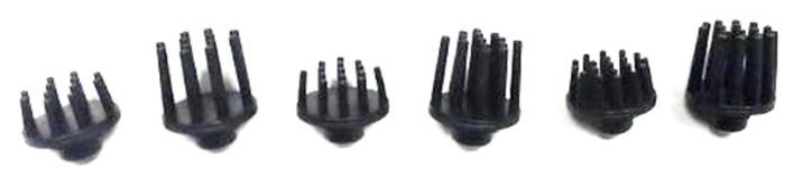

\subsection{Impedance measurements of various polymer compositions and electrode shapes}

Signal quality in biopotential recordings increases when lowering the contact impedance. Impedance measurements were carried out on electrodes with various conductive additives to optimize the composition. The impedance was measured on phantoms (platinum thin film and electrolyte wet cloth) to test composition reproducibility, and on human skin to evaluate the electrode-skin impedance variation between subjects. A potentiostat was used to generate constant voltage and measure the impedance of electrodes. Impedances are measured for DC up to $10 \mathrm{kHz}$, in plots only the impedance at $10 \mathrm{~Hz}$ is shown [5]. To perform a correct comparison, impedances are normalized towards skin area. 


\subsection{Mechanical properties of various polymer compositions}

Nanoindentation tests of the polymer electrodes with various carbon content were carried out using a nano indenter XP system (MTS Systems Corporation), equipped with a standard three-sided pyramid diamond indenter tip (Berkovich). From the experimentally obtained load-displacement curves, the elastic modulus $(E)$ and hardness $(H)$ are calculated based on their relationship to the contact area $(A)$ and the measured contact stiffness $(S)$ as indicated in next formulas:

$$
H=\frac{P_{\max }}{A} \quad S=\beta \frac{2}{\sqrt{\pi}} \sqrt{A}\left[\frac{1-v^{2}}{E}+\frac{1-v_{i}^{2}}{E_{i}}\right]^{-1}
$$

Where $P_{\max }$ is the maximum applied force, $A$ is the projected contact area of the indenter with the sample surface, $\beta=1.034, v$ is the poisson's ratio of the sample and $E_{i}(1140 \mathrm{GPa})$ and $v_{i}(0.07)$ are the elastic constants of the diamond indenter tip. Finally, the contact area $(A)$ was determined from the indenter tip shape calibration, as described in $[6,7]$.

\subsection{ECG/EEG recording and signal analysis}

The conductive polymer electrodes with the lowest impedance were used for signal recording. For strong signals such as ECG, passive electrodes were used. Wet and dry electrodes were placed next to each other on the skull of test subjects, and their signals were recorded simultaneously [8].

For signals with lower amplitude such as EEG $(10-100 \mu \mathrm{V})$, active electrodes were used for the conductive polymer electrodes to compensate for the slight higher impedance [9].

In this paper, a clinical EEG system was used for EEG signal recording. The reference and ground electrodes were mounted on the occipital area of subjects to avoid signal disturbance from eyes blinking. Wet and polymer dry electrodes were mounted on the central locations with $2-3 \mathrm{~cm}$ distance between each other to avoid gel smearing. During the recording, the subjects were asked to sit in a chair and relax with their eyes open or closed. With closed eyes, typically more 'alpha waves' are produced in the brain, having a frequency between 8 and $13 \mathrm{~Hz}$.

Correlation and coherence was calculated between signals obtained by wet and dry electrodes. In addition, the signal to noise ratio of the 'eyes-closed signals' was calculated, as described in [3].

\section{Results and Discussion}

\subsection{Conductivity optimization by impedance measurement}

In Fig. 2, the normalized impedance at $10 \mathrm{~Hz}$ is plotted for polymer electrodes with various carbon content, measured on phantoms and on the forearm skin of four different. The impedance on different subjects shows a significant variation from person to person. The impedance clearly reduces with increasing carbon content for both phantoms and skin. The impedance of the electrode with highest carbon content is $\sim 10$-fold higher than conventional wet electrodes on human skin.

The skin-electrode impedance decreases even more after skin pretreatments such as hydration, but these effects are only temporary [5]. For further ECG and EEG recording, the electrodes with 44\% carbon were used due to higher fabrication yield and lower hardness than those with $\sim 49 \%$ black. 
Figure 2. Normalized impedance at $10 \mathrm{~Hz}$ of conductive polymer electrodes with various carbon content on phantoms and forearm skin of four different subjects (S1-S4).

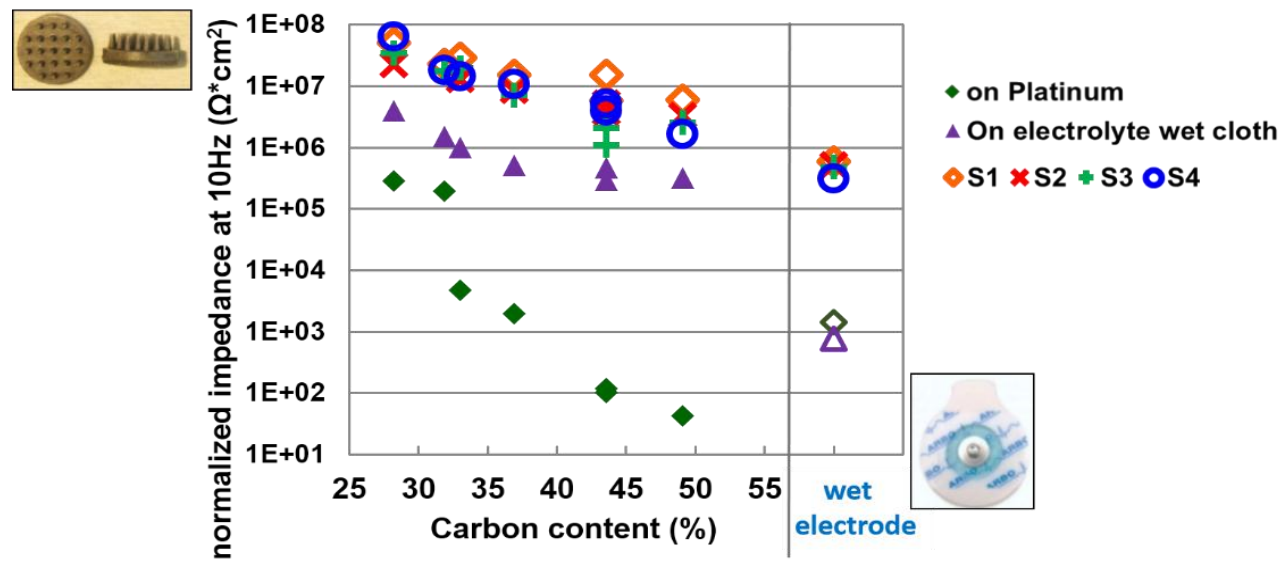

\subsection{Hardness and elastic modulus characterization by nanoindentation tests}

Nanoindentation tests were carried out on test cylinders of polymer with various carbon content. Hardness was determined using the loading cycle of the indentation test, while the elastic modulus was determined from the unloading cycle. Fig. 3 shows that the hardness and elastic modulus increases when carbon black content increases. This property limits the amount of carbon which can be added to the polymer matrix, since the harder the polymer, the lower the user comfort and the higher problems with polymer demolding (hence low fabrication yield) and brittleness. More results on mechanical properties as function of additive type and quantity will be published in [8].

Figure 3. Hardness and elastic modulus of polymer cylinders with various carbon content.
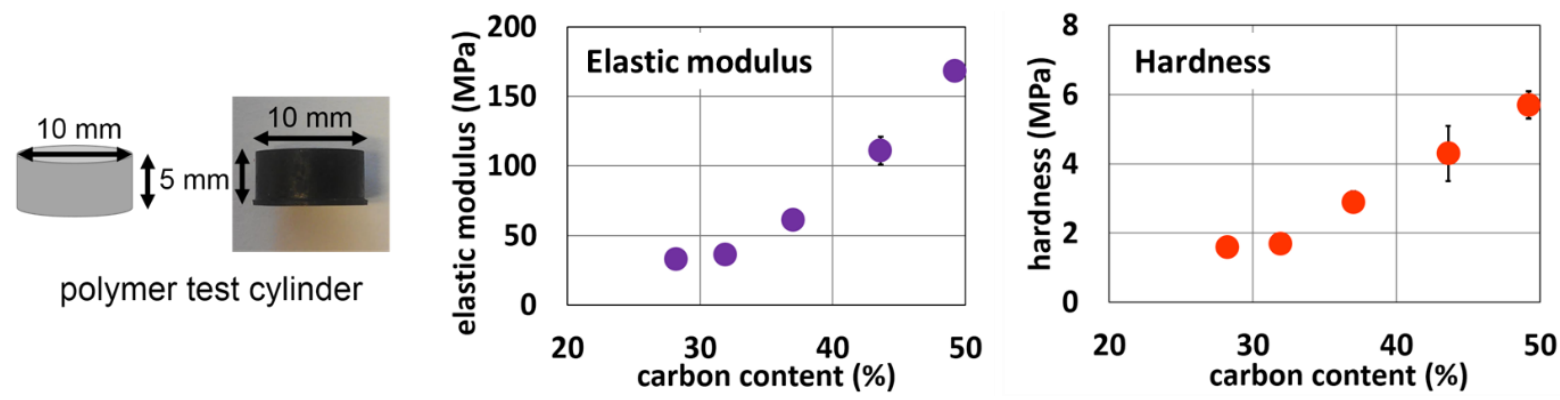

\subsection{ECG and EEG recording and signal analysis}

\subsubsection{ECG recording}

The ECG signals measured using wet and dry electrodes are very similar, see [5] for more details.

\subsubsection{EEG recording}

For an initial reference recording using only wet electrodes, the signals were named wetLL, wetL, wetR and wetRR. Then, a comparison recording with both wet and dry electrodes was performed, the signals were named wetL, dryL, dryR and wetR. The electrode locations are shown in Figs. 4 and 5.

For the reference EEG recording (all wet electrodes), signal correlation is shown in Fig. 4. As expected, the electrodes positioned closer to each other have higher correlation. Hence the wetLL-wetRR distance is the highest, the correlation the lowest. The ideal correlation of 1 is obtainable since it corresponds to 2 electrodes at exact the same location, covering a perfect identical brain region. 
Figure 4. The location of four wet electrodes and correlation of their signals.

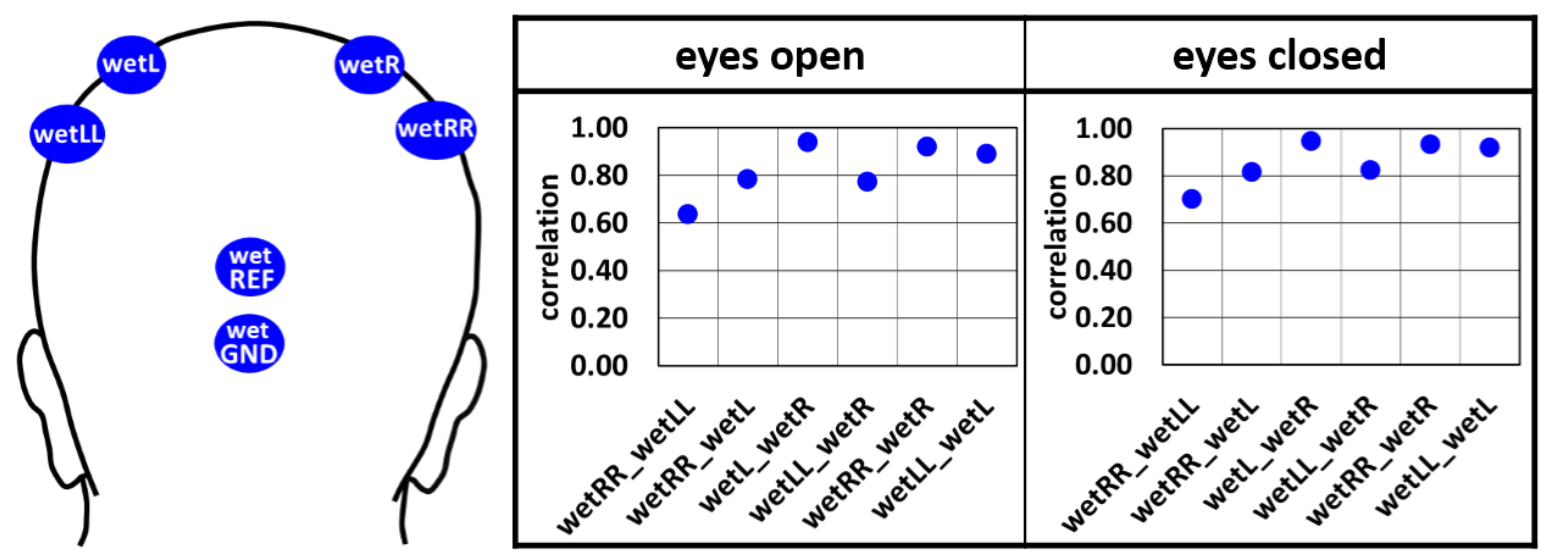

In the second experiment, the comparison recording, the polymer dry electrode was placed on the same location as wetL and got as labelling dryL (see Fig. 5). The EEG signals and frequency spectrum of wet and dry electrodes with the subject's eyes open and closed are shown in Fig. 5.

Figure 5. The EEG signal and spectrum of wet and polymer dry electrodes.

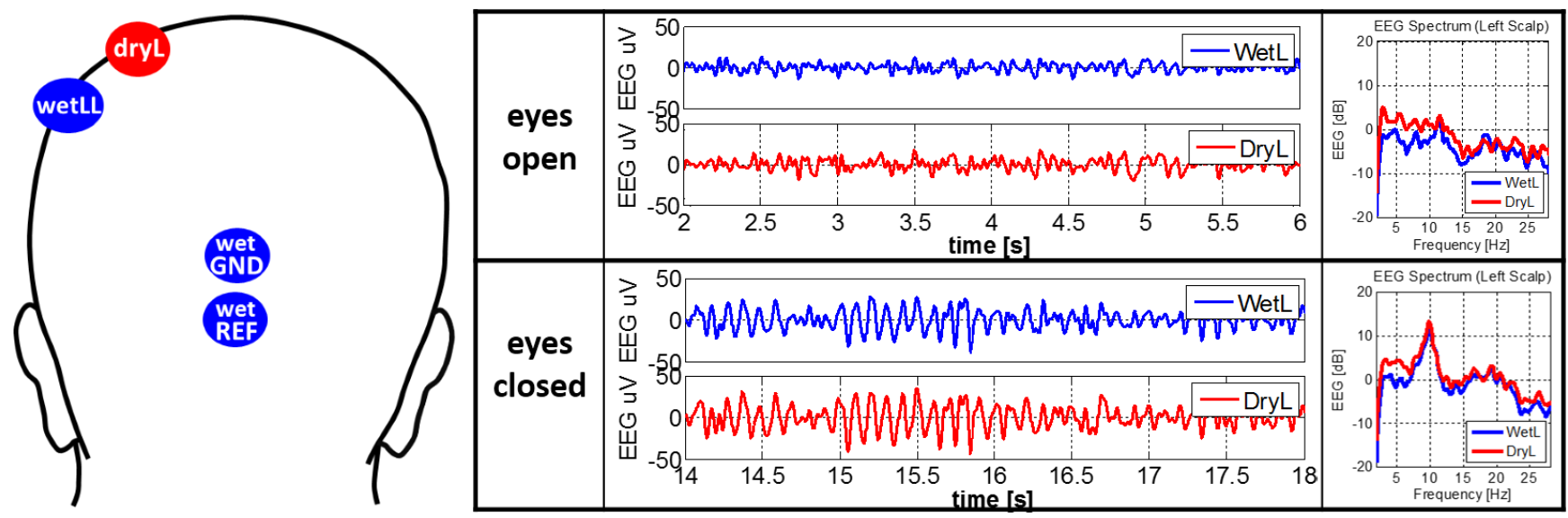

In Fig. 6, the signal correlation and coherence, and SNR of the closed-eye-signal are shown. The correlation and coherence of wet and dry electrode signals are very similar. For the SNR, the one dry electrode signal has a slightly lower SNR, but the alpha waves (the signal peak at $10 \mathrm{~Hz}$ ) can be detected very clearly (Fig 5).

Figure 6. The correlation, coherence of $8-13 \mathrm{~Hz}$ and SNR of wet and polymer dry electrodes

\begin{tabular}{|c|c|c|c|c|c|c|c|c|c|}
\hline \multicolumn{10}{|c|}{$\Delta$ wet electrode recording } \\
\hline \multicolumn{3}{|c|}{ correlation } & \multicolumn{2}{|c|}{ coherence $(8-13 \mathrm{~Hz})$} & \multicolumn{5}{|c|}{ SNR when eyes closed } \\
\hline 1 & & $A$ & 1 & & 10 & & & & \\
\hline 0.8 & $\Delta$ & & 0.8 & $\hat{\Delta}$ & 8 & $\boldsymbol{\Delta}$ & $\boldsymbol{\Delta}$ & $\Delta$ & \\
\hline 0.6 & & & 0.6 & & 6 & & & & $\bullet$ \\
\hline 0.4 & & & 0.4 & & 4 & & & & \\
\hline 0.2 & & & 0.2 & & 2 & & & & \\
\hline & open eyes & closed eyes & & closed eyes & & wet 1 & wet 2 & wet 3 & dry \\
\hline
\end{tabular}




\section{Conclusions}

Flexible polymer-based dry electrodes with high user comfort were fabricated. The polymer impedance decreases with increasing carbon content. The skin-electrodes impedance for $\sim 49 \%$ carbon content is about 10-fold higher than conventional wet electrodes. The hardness and elastic modulus both increase with higher carbon content. The polymer electrodes containing $\sim 45 \%$ of carbon were selected as best compromise between electrical and mechanical properties. ECG signals acquired from these optimum electrodes are very similar to the ones from wet electrodes. The EEG signals obtained by polymer electrodes are from high quality, with similar correlation and coherence as wet electrodesignals, and only a slightly lower SNR. Alpha waves can be observed clearly using both electrode types. All these results show that our polymer electrodes are very interesting alternatives for the rigid dry electrodes and the conventional gel electrodes.

\section{Conflicts of Interest}

The authors declare no conflict of interest.

\section{References}

1. Liao, L.-D.; Wang, I.J.; Chen, S.-F.; Chang, J.-Y.; Lin, C.-T. Design, fabrication and experimental validation of a novel dry-contact sensor for measuring electroencephalography signals without skin preparation. Sensors 2011, 11, 5819-5834.

2. Chi, Y.M.; Tzyy-Ping, J.; Cauwenberghs, G. Dry-contact and noncontact biopotential electrodes: Methodological review. IEEE Reviews in Biomedical Engineering 2010, 3, 1-1414.

3. Tautan, A.-M.; Mihajlovic, V.; Chen, Y.-H.; Grundlehner, B.; Penders, J.; Serdijn, W. In Signal quality in dry electrode eeg and the relation to skin-electrode contact impedance magnitude, International Conference on Biomedical Electronics and Devices, 2014; SCITEPRESS: pp 12-22.

4. Chen, Y.-H.; Op de Beeck, M.; Carrette, E.; Vanderheyden, L.; Grundlehner, B.; Mihajlovic, V.; Boon, P.; Van Hoof, C. In Polymer-based dry electrodes for high user comfort ecg/eeg measurements, Smart Systems Integration, Vienna, 2014; Vienna, pp 329-336.

5. Chen, Y.H.; Op de Beeck, M.; Vanderheyden, L.; Mihajlovic, V.; Grundlehner, B.; Van Hoof, C. In Comb-shaped polymer-based dry electrodes for eeg/ecg measurements with high user comfort, EMBC, 35th Ann. Intern. Conf. of IEEE, 3-7 July, 2013; pp 551-554.

6. Vanstreels, K; Urbanowicz A., Nanoindentation study of thin PECVD SiCOH low-k films modified in $\mathrm{He} / \mathrm{H}_{2}$ downstream plasma; J. Vac. Sci. Technol. B 28(1), 173-179

7. Oliver, W. C.; Pharr, G. M., In An improved technique for determining hardness and elastic modulus using load and displacement sensing indentation experiments, J. Mater. Res. 1992, 7, 1564-1583.

8. Chen, Y.-H.; Op de Beeck, M.; Vanderheyden, L.; Vandormael, H.; Vanstreels, K.; Van Hoof, C. In Soft conductive polymer dry electrodes for high-quality and comfortable ecg/eeg measurements, 13th Intern. Conf. on Modern Mat. \& Techn,; Trans Tech Publications: Montecatini Terme 2014.

9. MettingVanRijn, A.C.; Kuiper, A.P.; Dankers, T.E.; Grimbergen, C.A. Low-cost active electrode improves the resolution in biopotential recordings, EMBC, Bridging Disciplines for Biomedicine. Proc. 18th Annual Intern. Conf. of IEEE, 31 Oct-3 Nov 1996, 1996; pp 101-102 vol.101.

(C) 2014 by the authors; licensee MDPI, Basel, Switzerland. This article is an open access article distributed under the terms and conditions of the Creative Commons Attribution license (http://creativecommons.org/licenses/by/3.0/). 